\title{
Evidence for the Use of Complementary and Alternative Medicine for Pelvic Inflammatory Disease: A Literature Review
}

\author{
Dongmei Wang, ${ }^{1}$ Yue Jiang, ${ }^{1}$ Jiaxing Feng $\left(D,{ }^{2}\right.$ Jingshu Gao, ${ }^{1}$ Jinlan Yu, ${ }^{3}$ Jing Zhao, ${ }^{4}$ \\ Pihong Liu $\mathbb{D}^{1}{ }^{1}$ and Yaguang Han $\mathbb{D}^{1}$ \\ ${ }^{1}$ First Affiliated Hospital, Heilongjiang University of Chinese Medicine, Harbin, China \\ ${ }^{2}$ Heilongjiang University of Chinese Medicine, Harbin, China \\ ${ }^{3}$ Harbin Daoli District People's Hospital, Harbin, China \\ ${ }^{4}$ Heilongjiang Academy of Traditional Chinese Medicine, Harbin, China \\ Correspondence should be addressed to Pihong Liu; lph0804@163.com and Yaguang Han; haibin1421@126.com
}

Received 21 October 2021; Accepted 13 December 2021; Published 19 January 2022

Academic Editor: Yong Wang

Copyright (c) 2022 Dongmei Wang et al. This is an open access article distributed under the Creative Commons Attribution License, which permits unrestricted use, distribution, and reproduction in any medium, provided the original work is properly cited.

\begin{abstract}
Pelvic inflammatory disease (PID), a common infectious disease of the female reproductive tract, is mainly characterized by abdominal/pelvic pain and tenderness of the uterus, cervix, or adnexa on physical exam. In recent years, its incidence has gradually increased yearly due to numerous factors, including sexually transmitted diseases and intrauterine operations. Based on selfreport of PID in the National Health and Nutrition Examination Survey (NHANES) 2013-2014 survey, PID impacts approximately 2.5 million women in the US during their reproductive age. Although empiric treatments such as antibiotics or surgery could alleviate the related symptoms of PID, its unsatisfactory obstetric outcome and high relapse bring heavy physical and psychological burden to women. Complementary and alternative medicine (CAM), a complementary therapy other than Western medicine with a complete theoretical and practical system, has been attached to importance in the world due to its remarkable efficacy. More people are accepting and trying to use CAM to treat gynecological diseases, including infertility, polycystic ovary syndrome, and PID, but its efficacy and mechanism are still controversial. This article reviews the previous literature systematically focusing on the effectiveness, safety, and mechanism of CAM in the treatment of PID to provide an evidence-based basis for the clinical application of CAM in patients with PID.
\end{abstract}

\section{Introduction}

Pelvic inflammatory disease (PID) refers to the inflammation of the female organs located at the upper genital tract and their surrounding tissues (uterus, fallopian tubes, ovaries, parauterine tissues, and peritoneum) caused by pathogen infection, often involving adjacent tissues. Inflammation can involve one site or spread to several sites simultaneously, mainly including endometritis, salpingitis, tubo-ovarian abscess, and pelvioperitonitis, with the highest incidence rate of salpingitis $[1,2]$. The investigation in the recent ten years has shown that the prevalence of PID shows a significant upward trend. Approximately, $4 \%-12 \%$ of women during childbearing age worldwide suffer from PID [3]. In most developing countries, PID is more difficult to be controlled and effectively treated [4]. In China, the incidence rate of CPID in women who have more than 5 years of intercourse exceeds $20 \%$ [5], which seriously affects women's physical and mental health. From July 2013 to March 2014, a survey of 1010 women in a certain area of China showed that the prevalence rate of CPID was $5.8 \%$ and pointed out that abortion was one of the crucial factors of PID infection [6]. A survey of 1100 patients in a hospital found that the proportion of patients with reproductive tract infection was $4.36 \%$, of which PID constituted $14.6 \%$ [7]. A census for diseases of married women in rural areas in China showed that the prevalence of PID accounted for $13.5 \%$ among all diseases on average, and in some areas, it could be highly $23.53 \%$ [8]. 
Neisseria gonorrhoeae and Chlamydia trachomatis are the main pathogenic microorganisms. In addition, some aerobic bacteria, anaerobic bacteria, viruses, and mycoplasma also participate in its occurrence. Pathogenic microorganisms are mostly mixed infections ascending from the vagina, leading to local tissue congestion, edema, inflammatory exudation, connective tissue hyperplasia, followed by irregular menstruation, abnormal leucorrhea, etc. $[9,10]$. On most occasions, the symptoms of PID vary from none to severe. PID can be divided into two categories, namely acute PID and chronic PID. Chronic pelvic inflammatory disease (CPID), caused by PID not receiving prompt and effective treatment, can be accompanied by inflammatory lesions such as pelvic adhesions and tubal obstruction, leading to infertility, ectopic pregnancy, and chronic pelvic pain [11]. Due to the great variation of the clinical manifestations, the diagnosis of PID could not be completely accurate [12]. The diagnostic criteria recommended by the Centers for Disease Control and Prevention (CDC) are lower abdominal or pelvic pain and at least one of the following: adnexal tenderness or cervical motion tenderness or uterine tenderness [13].

PID has the characteristics of the long course, low cure rate, and high recurrence rate. Empirical therapies involves broad-spectrum combination regimens of antimicrobial agents to cover likely pathogens, and surgery can be performed if necessary [14]. Although the incidence and severity of PID in North America and Western Europe had reduced by using antibiotics in the past two decades, the ultimate efficacy was still not satisfactory. Complementary and alternative medicine (CAM), a supplement to conventional medicine, mainly includes the following methods: alternative medical systems, physical and mental intervention, biological therapy, manipulation and body-based methods, energy therapy, etc. [15]. The relevant report suggested that the rate of CAM application has reached $9.8 \%-76.0 \%$ globally [16]. At present, CAM has been widely utilized in female genital infections. Several randomized controlled trials (RCTs) have found that CAM has antibacterial and anti-inflammatory therapeutic effects, which could be effective in treating PID [17], but its efficacy and mechanism still exist in dispute. This article summarizes the efficacy of Chinese herbal medicine (CHM), acupuncture and moxibustion, pelvic exercises, hyperbaric oxygen therapy, and microwave physiotherapy in the treatment of PID and further explores their possible mechanism and safety in treating PID.

\section{Researching the Overview of CHM in Treating PID}

$\mathrm{CHM}$, an integral part of traditional Chinese medicine (TCM), has a long history of treating diseases. In ancient times, Chinese people used certain animals, plants, and minerals to relieve the symptoms of diseases. Through longterm practice, the theories and methods of TCM have gradually formed and are recorded in written works, such as The Yellow Emperor's Inner Classic, Treatise on Cold Damage, and A Hundred Records on "Shen Nong's Classic of the Materia Medica". Its basic characteristics are the concept of holism and syndrome differentiation and treatment. TCM has been widely used in different stages of disease, such as prevention, diagnosis, treatment, and rehabilitation. With the development of science and technology, TCM staff have provided a more theoretical basis for TCM to treat diseases by combining modern technology with original theories [18]. CHM has various dosage forms (e.g., pills, powders, granules, wines, tinctures, and ointments). It can also be processed into Chinese patent medicines. PID always threatens women, which needs to attract people's attention. For patients suffering from PID, adjuvant treatment of CHM seems to be in high demand. Studies have shown that CHM mainly administered by oral and retention enema has a definite curative effect and obvious advantages in treating gynecological diseases, especially for PID [19].

\subsection{Clinical Effects of Oral Chinese Medicine Compound} Formula (CMCF) in Treating PID. Clinically, oral CMCF combined with conventional therapy is used to treat PID. More and more RCTs showed that the combination therapy could greatly improve the clinical effective rate and reduce adverse reaction and recurrence rate [20]. Zhang and Zhang [21] found that CMCF combined with antibiotics in treating acute PID can shrink pelvic mass; reduce pelvic fluid, inflammatory factors, and adverse reactions; and improve immune function. Xiangli took the same method to treat PID. After treatment, the patients' symptoms such as fever, lower abdominal pain, and abnormal leucorrhea were significantly improved [22]. Feng's research showed that CMCF combined with antibiotics could effectively decrease the time of symptom relief and reduce the TCM syndrome score and the deepest diameter of pelvic effusion [23]. Wang and Gan added another CMCF (treatment arm) to treat PID based on CMCF combined with antibiotics (control arm) and found that the cure rate of treatment arm was higher than that of the other arm $(P<0.05)$. After treatment, TCM syndrome score, inflammatory mass diameter, pelvic effusion depth, serum ICAM-1, D-dimer, plasma viscosity, erythrocyte sedimentation rate, and platelet aggregation rate of treatment arm decreased more significantly than those of control arm. IL-2, IL-10, MMP-2, complement C3, and C4 were significantly higher than those of control arm [24]. Xiong et al. [25] also found that CHM combined with antibiotics has a more obvious effect on CPID. After treatment, the number of leukocytes, the proportion of neutrophils, and CD8+ and Th2 in treatment arm were significantly lower than those in the control group $(P<0.01)$; the levels of CD3+, CD4+, CD4+/CD8+, Th1, and Th1/Th2 were significantly higher than those in the control group, and the total effective rate was significantly higher than that in the control group $(P<0.05)$. Xiong [26] found that the serum estradiol (E2), cancer antigen 125 (CA125), and hemorheological indicators of PID patients treated with CMCF combined with antibiotics were lower than those treated with antibiotics alone. Besides, some studies have reached the same conclusion [27-30]. In a double-blind, multicenter, placebo-controlled clinical trial, 155 patients 
with PID were randomly assigned into treatment arm $(n=77)$ and control arm $(n=78)$. The treatment arm was given CMCF combined with antibiotics placebo in the first two weeks. Subsequently, the patient received oral CMCF merely for the remaining weeks. While the control arm was administered two kinds of medicines together with CMCF placebo in the first two weeks and CMCF placebo for the remaining weeks. The follow-up results after treatment showed that the cure rate together with the effective rate of TCM syndrome in treatment arm was $81.82 \%$, significantly higher than $67.95 \%$ in control arm $(P<0.05)$, which demonstrated that the oral CMCF could reduce the antibiotic dosage required for PID treatment and improved symptoms in PID patients [31]. In addition, some studies are comparing the efficacy of oral CMCF with conventional therapy. Zhou and Chen [32] compared the efficacy of CMCF with antibiotics in treating endometritis by observing the morphological changes of the endometrium through hysteroscopy and performing an endometrial biopsy at a fixed position. The results showed that the markedly effective rate of clinical symptoms in the CMCF group was $91.3 \%$, significantly higher than that of the control group $60.0 \%$ $(P<0.05)$, and plasma cell CD38 infiltration of the endometrial stroma was significantly decreased, the positive expression of MUC-1 increased, and the expression of HIF$1 \alpha$ was reduced, indicating that CMCF has a better therapeutic effect on chronic endometritis compared with antibiotics. The clinical effects were also not completely consistent among different oral CHMs. Wu et al. compared the clinical efficacy of two CMCFs (Tongluo Qingre Decoction and Gexia Zhuyu Decoction) on PID through an RCT and found that the effective rate of Tongluo Qingre Decoction was higher than that of Gexia Zhuyu Decoction $(P<0.05)$. After treatment, compared with the patients administered with Gexia Zhuyu Decoction, those taken Tongluo Qingre Decoction had lower TCM syndrome scores, and the patients' peak systolic flow velocity of uterine blood flow levels was higher $(P<0.05)$, and the flow resistance index and pulsatility index were lower $(P<0.05)$ than those administered with Gexia Zhuyu Decoction. The study concluded that Tongluo Qingre Decoction had better efficacy in treating PID and was more conducive to improving uterine blood microcirculation [33]. Huang randomized 180 CPID patients to CMCF arm, physiotherapy arm, and combination therapy arm. Patients in CMCF arm were given oral CMCF, physical therapy arm was given physical therapy, and combined treatment arm was given oral CMCF combined with physical therapy. After treatment, the effective rate of combined therapy arm was $98.3 \%$, which was significantly higher than that of CMCF arm (86.7\%) and physical therapy arm (76.7\%), thus demonstrating that oral CMCF combined with physiotherapy could significantly improve the effect of CPID patients, strengthen the immunity of patients, and reduce inflammation. In addition, it can improve blood rheology indicators as well [34]. Furthermore, studies have found that the combination of CMCF and antibiotics for PID is not as good as expected. Lan et al. combined CMCF with antibiotics to treat CPID. The total effective rate of treatment arm was $95.00 \%$.
Although the levels of C-reactive protein and IL-10 were significantly lower than those in control arm after treatment, the incidence of adverse reactions and recurrence rate at 3 and 6 months were similar, without any significant difference [35]. Table 1 lists some of the above study protocols and some unmentioned protocols [24-38].

\subsection{The Mechanism of Oral CMCF in Treating PID.} Extensive research has proved that the mechanism of $\mathrm{CHM}$ in treating PID is associated with inflammatory cells, inflammatory factors, and related pathways. Zhang et al. found that the administration of CHM to CPID-like rats could decrease the levels of IL-2, IL-6, IL-10, TNF- $\alpha$, and TGF- $\beta 1$ in serum, increased the mRNA of P53, Fas/FasL, and MMP2 mRNA in the uterus, while decreased TGF- $\beta 1$ mRNA, and inhibited NF- $\kappa \mathrm{B}$ p 65 in the uterus and ovary tissue. These results indicated that the possible mechanism of $\mathrm{CHM}$ in treating CPID was achieved by inhibiting the inflammatory response, inducing the inflammatory cell apoptosis, and downregulating serum inflammatory cytokines [36]. A study conducted by Zou et al. [37] has suggested that CHM could inhibit the infiltration of lymphocytes and neutrophils in the fallopian tubes of PID-like rats, reduce the release of IL- $1 \beta$, IL-6, IL-8, and MCP-1, promote the production of LXA4, and found that CHM could regulate LPS-stimulated NF- $\kappa \mathrm{B}$ signaling activity and promote FPR2 expression in THP-1 cell line, therefore contributing its anti-inflammatory effect. The experimental results of Zheng et al. showed that compared with PID-like rats not administered with CHM, the levels of serum IL- 6 , IL- 8 , and TNF- $\alpha$ in PID-like rats treated with $\mathrm{CHM}$ significantly decreased. It was concluded that CHM had strong anti-inflammatory and anti-infective effects, and could be used to treat PID and relieve pain [38]. Sun [39] explored the efficacy of CHM combined with levofloxacin in treating CPID. 120 patients were randomized into control arm and observation arm. The control arm was given levofloxacin lactate injection, and the observation arm was combined with CHM on the basis of control arm. After treatment, the levels of IL-2 and IL-10 in the observation arm were higher than those in the control arm, and the levels of CRP and TNF- $\alpha$ were lower than those in the control arm $(P<0.05)$. Xia et al. [40] analyzed endogenous small-molecule metabolites in the serum of rats after CHM treatment based on gas chromatography-mass spectrometry (GC-MS)based metabolic profiling method combined with multivariate statistical analysis, such as PCA, PLS-DA, and OPLSDA. The results showed that CHM treatment could significantly improve the inflammatory pathological characteristics and tissue damages of model rats. Based on the principle of VIP $>1$ and $P<0.05$, six different metabolic biomarkers, i.e., L-valine, L-isoleucine, L-threonine, butanedioic acid, serine, and D-glucose, were identified, and their contents were significantly reversed after administration. Further analysis of the metabolic pathways in the KEGG database showed that CHM could achieve this effect through the metabolism of glycine, serine, and threonine, biosynthesis of aminoacyl tRNA, and biosynthesis of valine, leucine, and isoleucine. $\mathrm{Li}$ et al. [41] used the network 


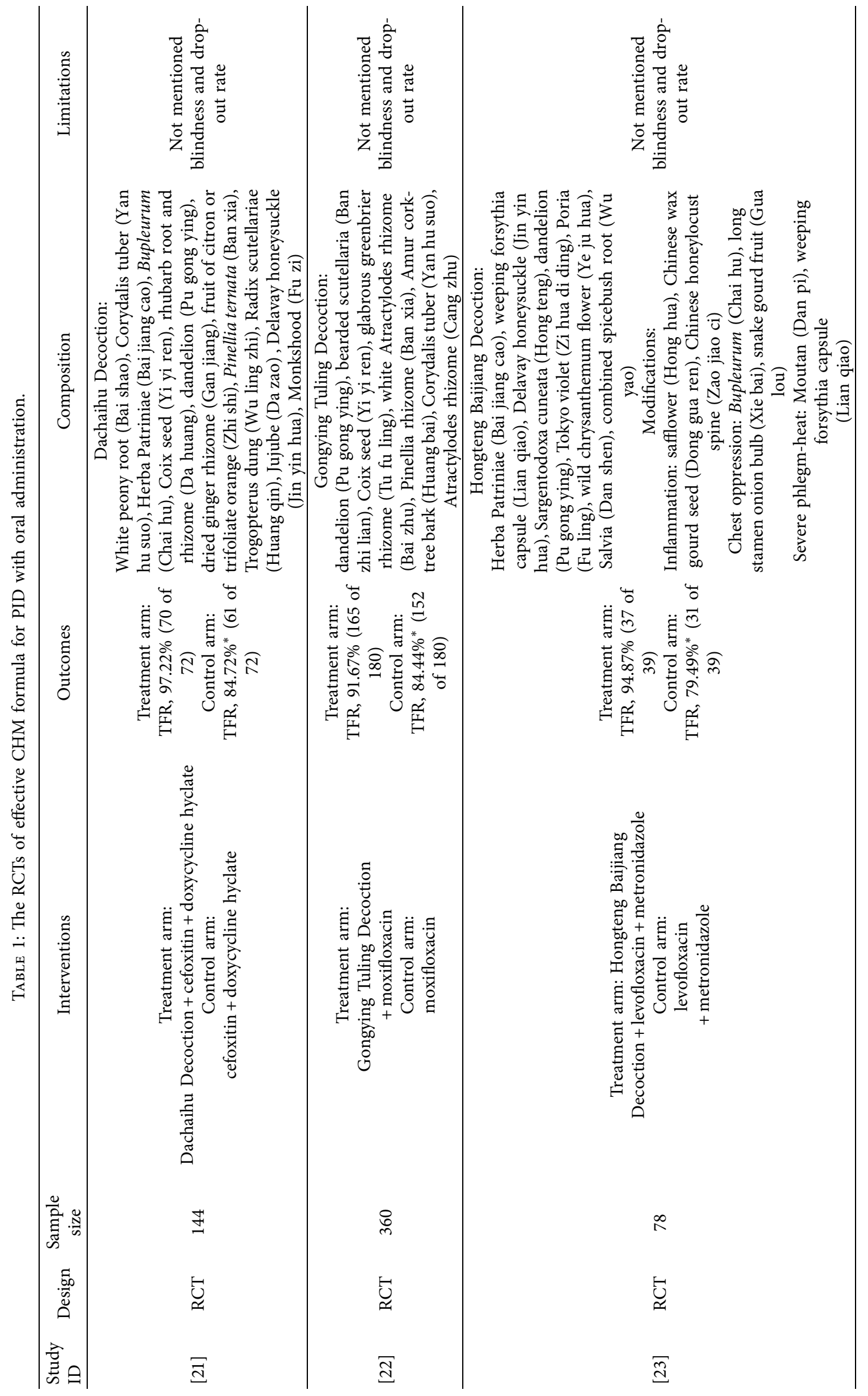




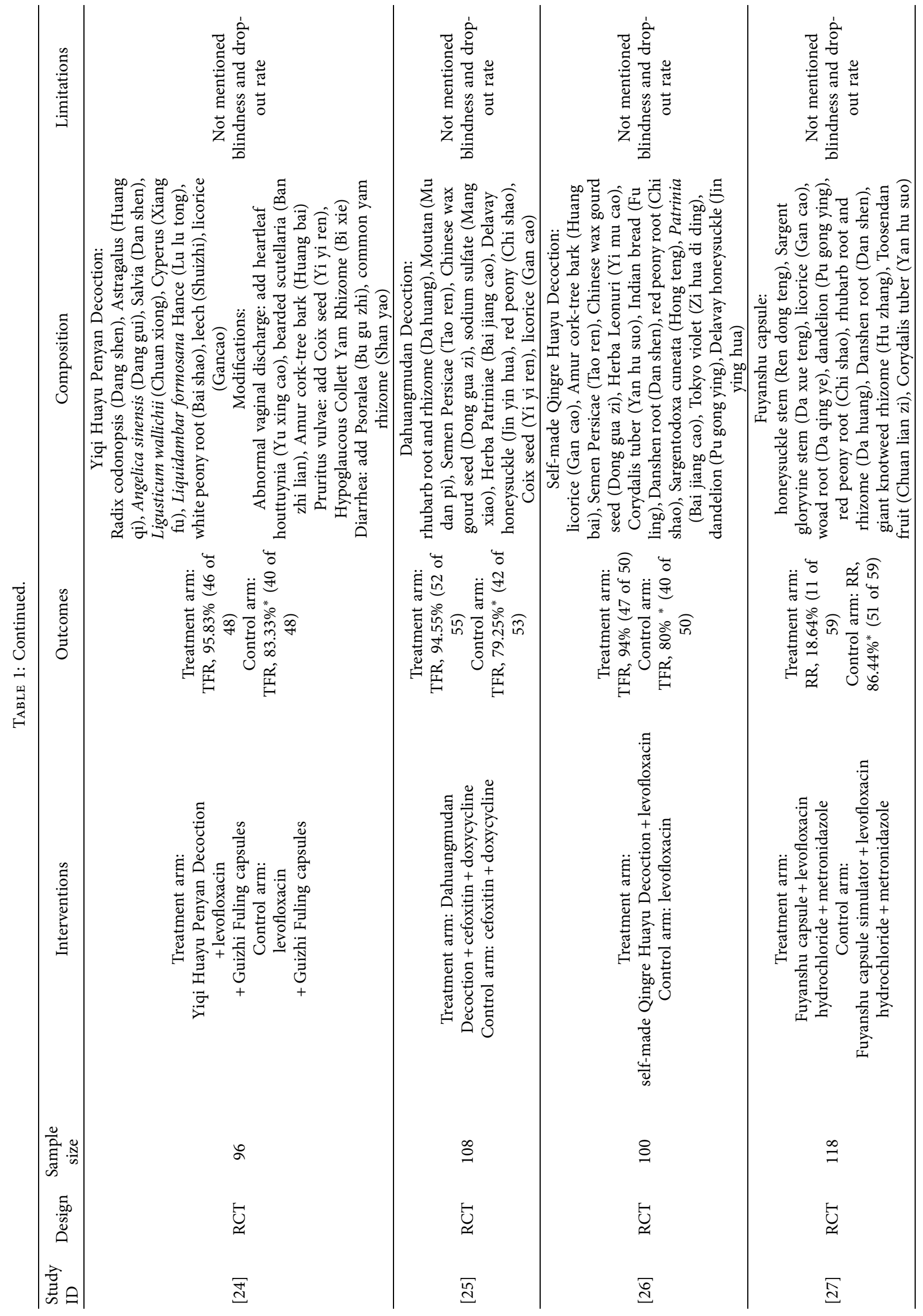




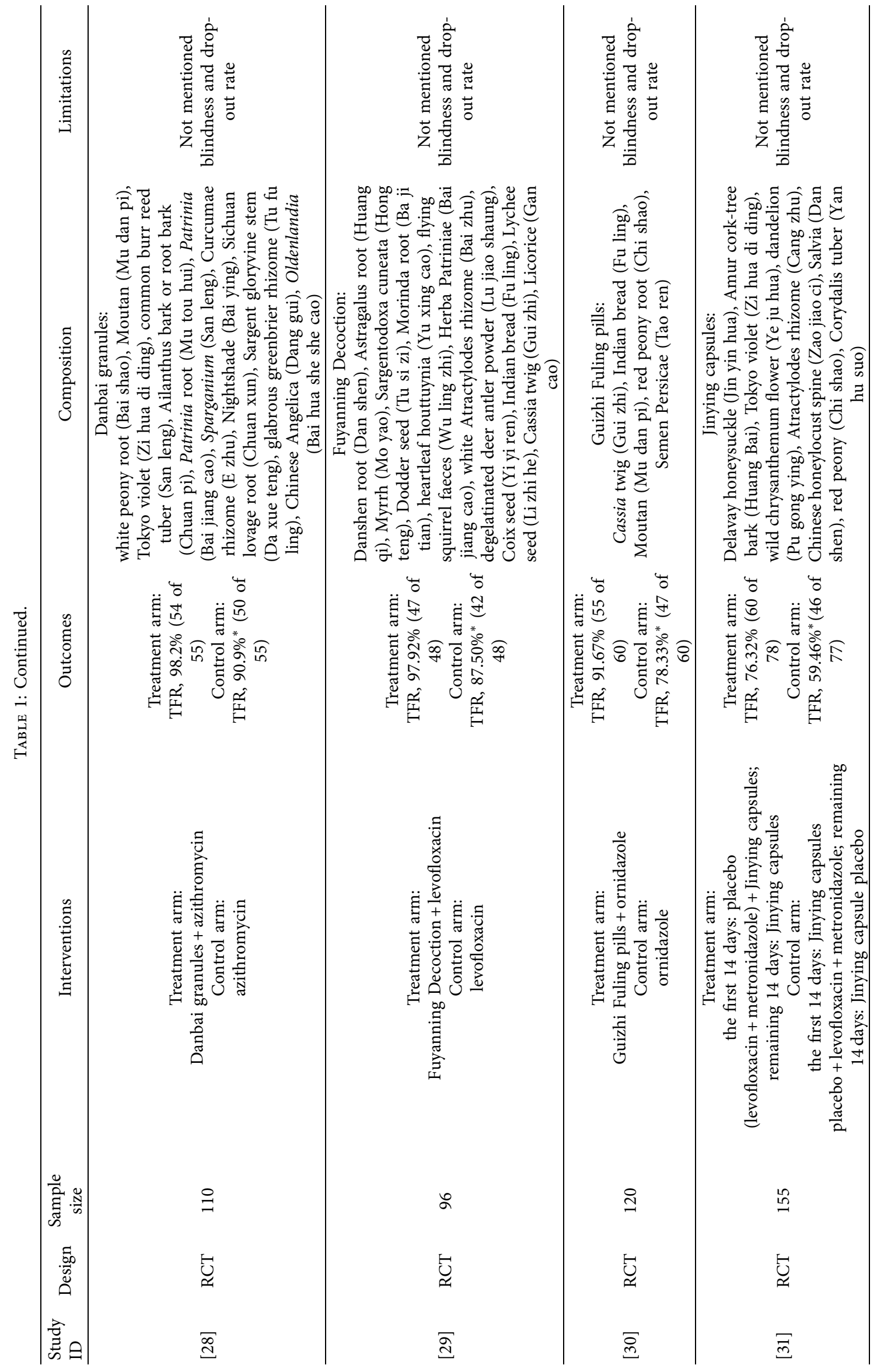




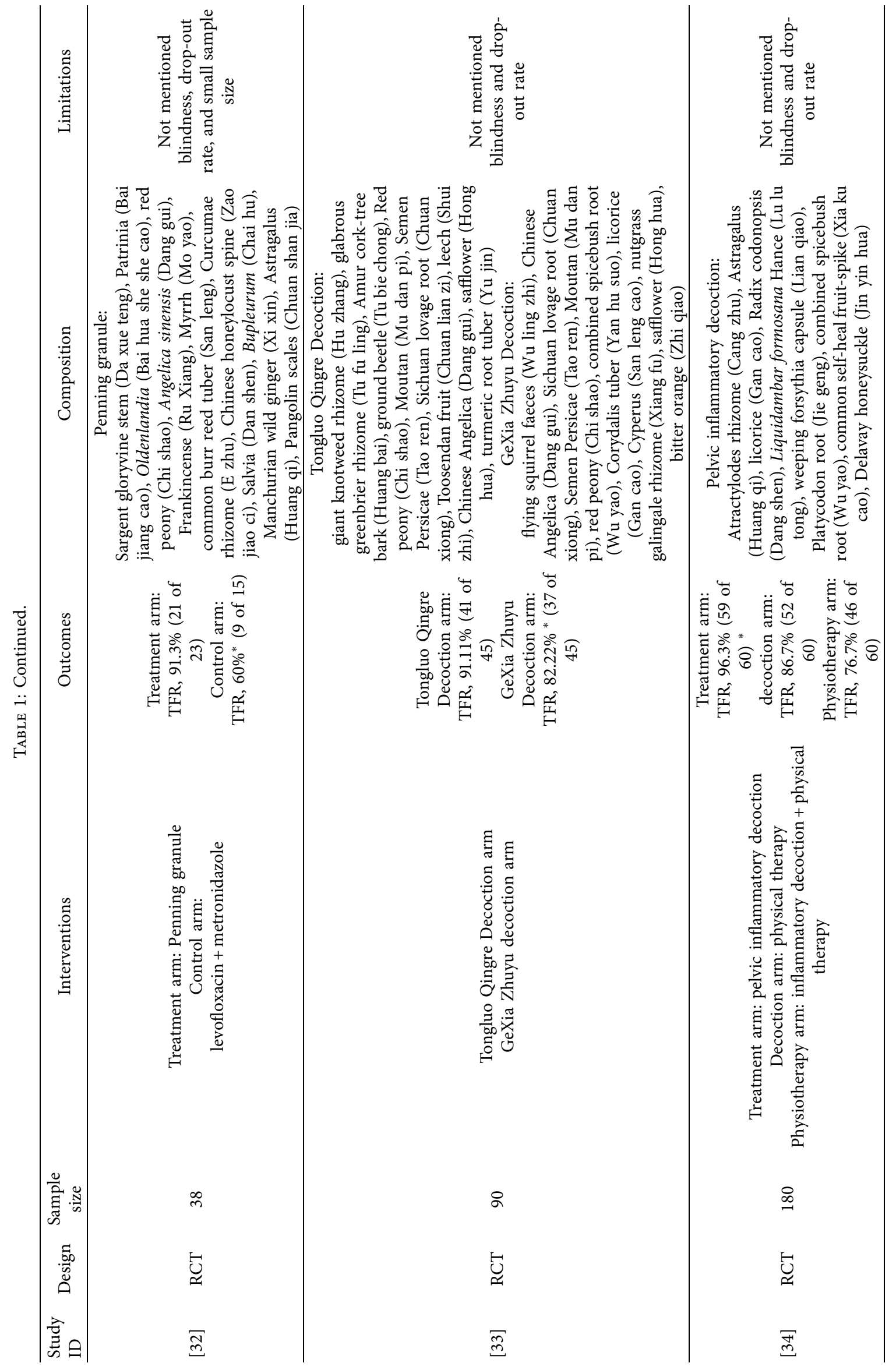




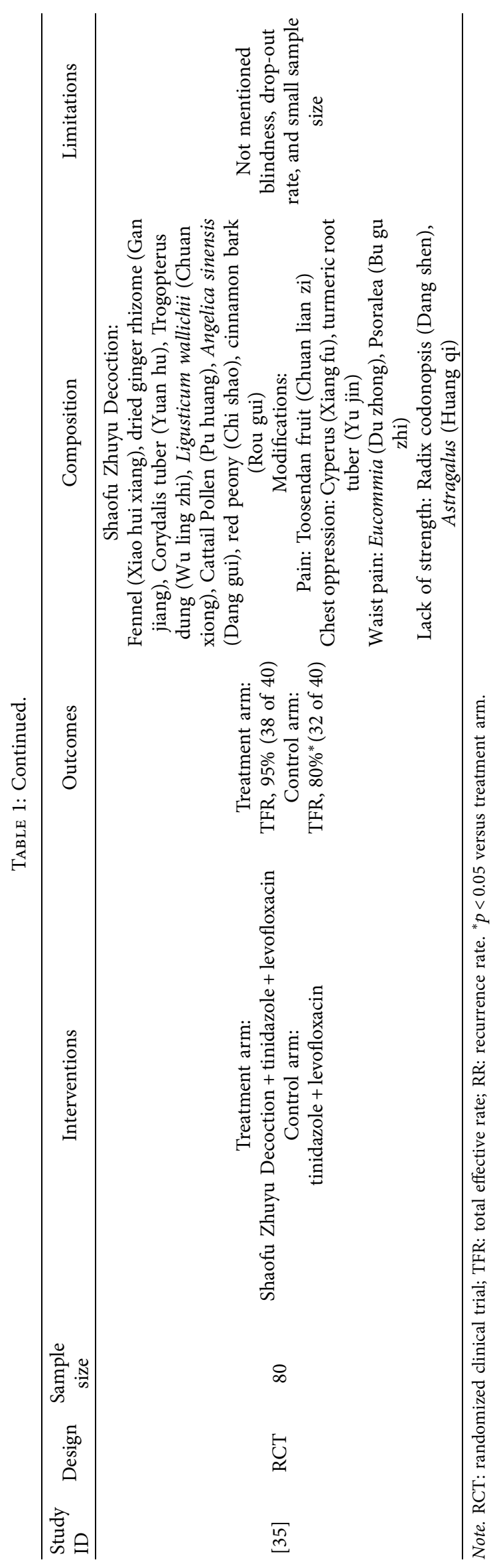


pharmacology method to screen targets and found that the PTGS2 target in the arachidonic acid (AA) pathway was significantly correlated with CPID, which further confirmed that CHM could reduce the development of CPID by regulating PTGS2 target.

\subsection{Clinical Effects and Mechanism of CHM Retention Enema} (CHMRE) in Treating PID. CHMRE evolved from the enema method in "Treatise on Cold Damage" is one of the most commonly used external treatment methods and also known as anorectal administration. Pouring CHM into the rectum and keeping it for four to five hours to be absorbed fully by intestinal mucosa to treat the disease. In line with physiological and anatomical characteristics of the female pelvic cavity, the transrectal administration of CHM can make the drug absorbed through the mucosal venous plexus [42-44], directly arrive at the lesion, accurately and quickly exert the drug effect, improve local microcirculation, and promote the absorption of effusion and mass $[45,46]$. The possible mechanism of CHMRE in treating PID is related to its ability to improve blood rheology, reduce oxidative stress response, reduce inflammation, etc. [47]. Due to its simplicity and convenience, CHMRE has a wide range of clinical applications. In addition, compared with conventional therapies, the medicine enters the internal iliac vein through the inferior rectal vein and/or anal vein, then finally into the systemic circulation, which can reduce the irritation of the digestive system, avoid the first pass elimination to improve the bioavailability of drugs, and reduce the damage to the liver and other organs, therefore greatly reducing the adverse effects and side effects [48-50]. To assess the efficacy of CHMRE in treating PID, Liang and Ling carried out a comparative study on 184 patients who suffered from sequelae of PID and found that the total effective rate of CHM staining therapy combined with CHMRM arm was higher than that of abdominal ultrasound drug delivery therapy arm, with statistically significant difference $(P<0.05)$ [51]. A clinical study [52] on a larger number of people has reported that compared with 55 patients with routine anti-inflammatory treatment, patients with CHMRE combined with anti-inflammatory treatment had a significantly higher effective rate, and serum TNF, IL-2, and IL-10 levels were statistically significant. Research conducted by Shao demonstrated that the CHMRE has a significant effect on PID, especially in terms of pain relief, recurrence reduction, and prevention of long-term complications [53]. More recently, numerous clinical studies have drawn the same conclusion [54-56]. Some researchers utilized CHMRE after hysteroscopy, which effectively reduced the levels of TNF- $\alpha$, IL-6, and IL-8, improved the unobstructed fallopian tube, and relieved abdominal pain and other symptoms, thereby promoting the recovery of fertility [57-60]. CHMRE could also reduce the recurrence rate of PID for half a year or more $[61,62]$. Based on the above-mentioned research works, CHMRE has significant efficacy in treating PID with a low incidence of adverse reactions, which is worthy of clinical application. However, it should be noted that the temperature of $\mathrm{CHM}$ should be between 39 and $41^{\circ} \mathrm{C}$ to prevent intestinal spasms or scald of intestinal mucosa caused by inappropriate temperature. Of course, CHMRE also has its shortcomings. For very few patients, the intolerance of it manifested as severe diarrhea and abdominal distension affects their quality of life seriously. In addition, for patients after intestinal tumor surgery, since the normal barrier function of the intestine has been broken, special attention should be paid to the medication. The above studies are listed in Table 2.

\subsection{The Application of Chinese Medicine Monomer in Treating} PID. Apart from CMCF, Chinese medicine monomers are also generally used in treating PID. Sargent gloryvine stem (SC) and Patrinia scabiosifolia (PS) have the effects of clearing heat and resolving toxins, invigorating blood, and dissolving stasis, which are the key to treating PID. Modern pharmacological studies have suggested that SC and PS contained various active ingredients such as phenols, flavonoids, phenylpropenes, and triterpenes, which have antioxidant, antibacterial, anti-inflammatory, and antiviral effects [63]. Their active ingredients act on key targets such as VEGFA, VWF, IL6, TNF, and NFKB1, therefore regulating AGE-RAGE, FA, Toll-like receptors, PI3K/Akt, NF$\kappa \mathrm{B}$, apoptosis, and cancer signaling pathways. Zhang $\mathrm{Y}$ used Chinese medicine Smilax china L. to treat PID. Its active ingredient Smilax china polysaccharide is considered to have an anti-inflammatory effect. Through extraction, purification, and structural identification, it was discovered for the first time that Smilax china L. polysaccharide can be purified to produce polysaccharides SCLP1 (Smilax china L. polysaccharide $1,42.1 \mathrm{kDa}$ ) and SCLP3-2 (Smilax china L. polysaccharide $3,2,16.8 \mathrm{kDa}$ ), which structure had been identified by chemical and spectral analyses. The results showed that SCLP1 and SCLP3-2 could inhibit the production of NO and IL- 6 in RAW264.7 cells stimulated by LPS through NF- $\kappa \mathrm{B}$ and MAPKs (ERK1/2, JNK) pathways [64]. Kong D studied the therapeutic efficacy and potential mechanism of Asian acid (AA) on PID-like rats. The results showed that AA treatment significantly reduced the overproduction of cytokines and chemokines and inhibited MPO activity, NLRP3 inflammasome, activation of NF- $\kappa \mathrm{B}$ and caspase-3, and oxidative stress, indicating that AA had stronger anti-inflammatory and antioxidant effects on PIDlike rats. Its anti-inflammatory mechanism may be related to the inhibition of NLRP3 inflammasome activity and the NF$\kappa \mathrm{B}$ pathway [65].

\section{Researching the Overview of Acupuncture and Moxibustion in Treating PID}

Acupuncture and moxibustion, a widely practiced traditional medical system that existed for more than 3,000 years, is considered to be rooted in naturalistic theories compatible with Confucianism and Taoism [66-68]. Since the reform and opening up, acupuncture and moxibustion have been gradually accepted by Western countries, which have been playing an important role in the internationalization of TCM. With its unique advantages (e.g., simple operation, 


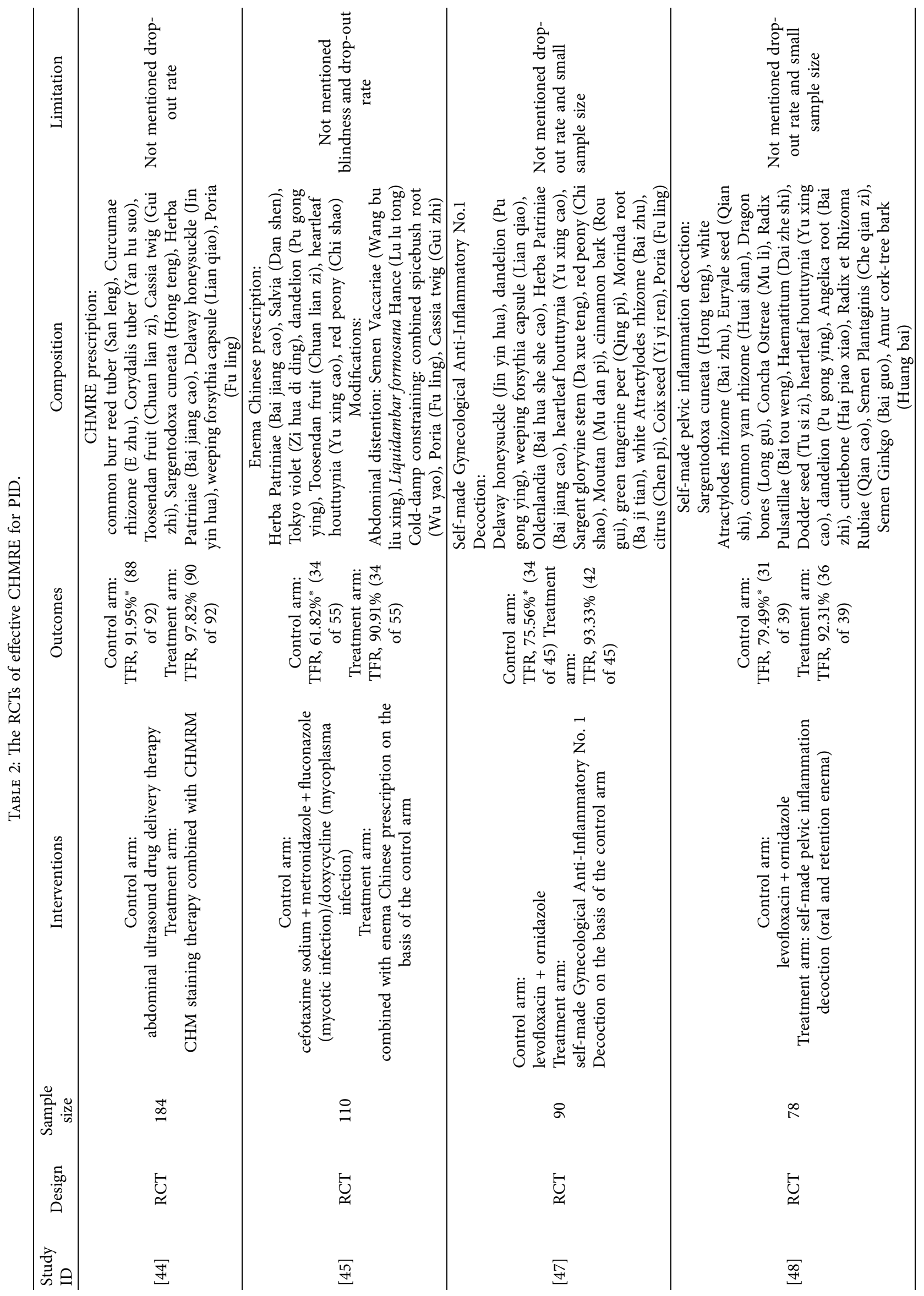




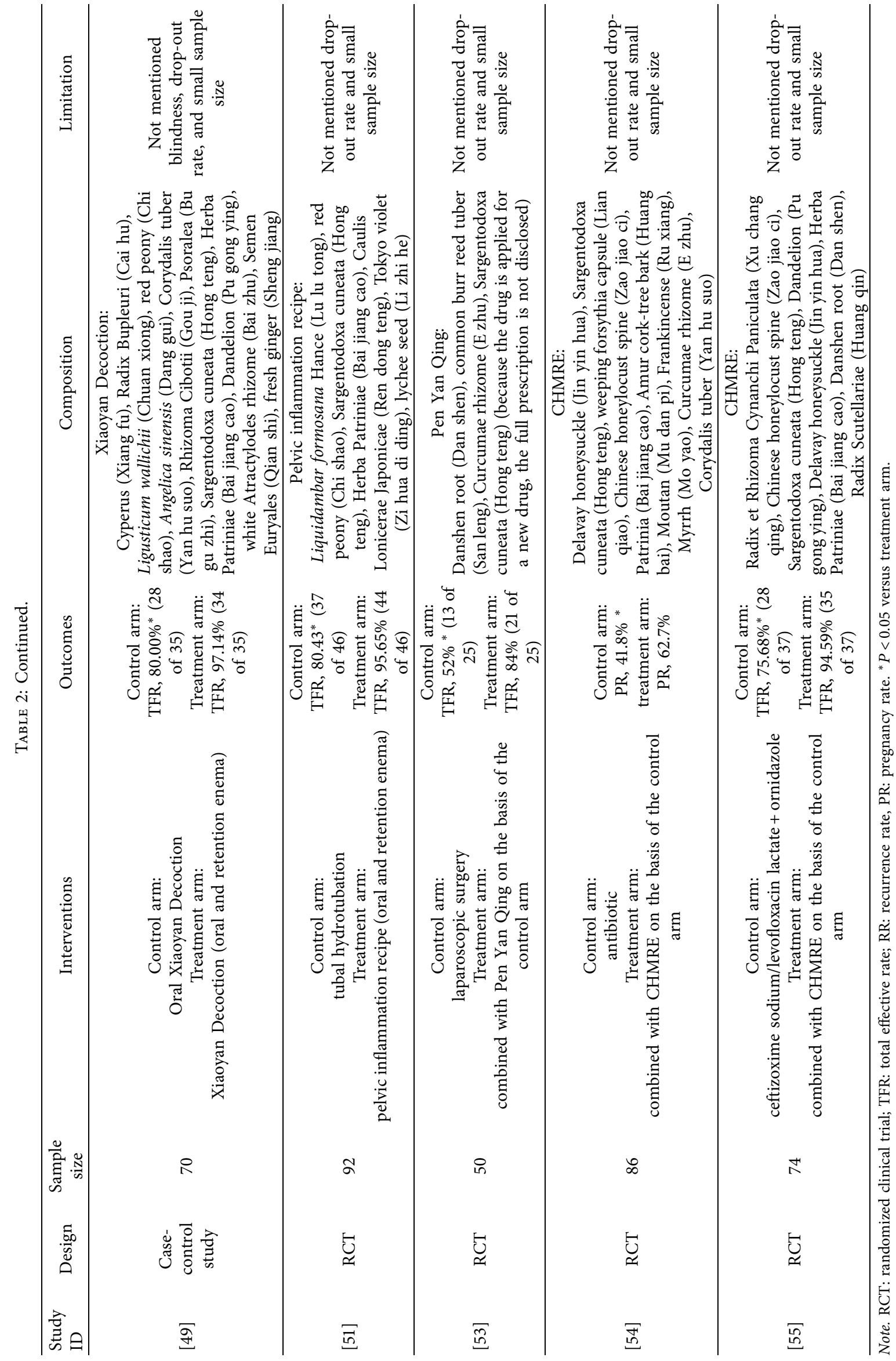


durable, and strong quantity of stimulus), acupuncture and moxibustion have been recognized and applied in 183 countries around the world. There are about 200,000 practitioners [69-73]. In clinic, acupuncture and moxibustion are usually divided separately. Guided by the theory of meridians and acupoints, practitioners use needles and artemisia as tools and materials to stimulate specific parts of the body through needles or burning artemisia to adjust the balance of Yin-Yang, therefore achieving the purpose of treating and preventing diseases. PID usually has longer treatment courses and a relatively low cure rate due to its complicated etiology. As a branch of CAM, acupuncture can enhance immunity and relieve the lower abdominal pain of PID patients. Moreover, it is simple in operation, available, and accepted by patients because of its fewer side effects. Due to such superiorities, acupuncture and moxibustion have aroused the attention of many gynecologists, so a series of clinical and animal studies have been carried out to evaluate the effect and therapeutic mechanism in PID.

3.1. Clinical Effect of Acupuncture in Treating PID. Acupuncture can effectively treat more than 500 types of diseases. In 1971, the acupuncture anesthesia test was successful, which set off an upsurge of acupuncture anesthesia. Later, when US President Nixon visited China in 1972, the accompanying journalists experienced acupuncture for analgesia, which made medical workers worldwide arouse great interest in acupuncture for treating diseases [74, 75]. Acupuncture has a long history of treating gynecological diseases, and abundant studies have shown that acupuncture is beneficial, particularly in treating CPID $[76,77]$. There are many categories of acupuncture treating CPID, including filiform acupuncture, warming-acupuncture, etc. Selecting appropriate acupuncture based on patients' clinical manifestations is conducive to their recovery [78]. Before the 1980s, there were fewer reports on the related studies about acupuncture in PID, let alone the large-sample clinical RCTs. In 1989, Wang [79] randomly assigned 95 patients with CPID into treatment arm (electroacupuncture (EA) combined with moxibustion) and control arm (antibiotic). The results showed that the therapeutic effect of EA combined with moxibustion was better than antibiotics $(P<0.05)$. This was the first clinical trial to show that acupuncture was effective in treating PID. Subsequently, in 2008, Zhen and Wang [80] randomized 85 CPID patients into the warmingacupuncture arm and the CHM arm and concluded that the effective rate was $95.6 \%$ versus $77.5 \%$, respectively, with a significant statistical difference $(P<0.05)$. During the follow-up, the recurrence rate of the treatment arm was $8.7 \%$, while the other arm was $22.2 \%$. Zhang [81] compared the efficacy of Western medicine with acupuncture combined with CHMRE in treating patients with acute PID. The results showed that the effective rate of acupuncture combined with CHMRE was higher than that of Western medicine $(P<0.05)$. Therefore, it is concluded that acupuncture combined with CHMRE is effective in treating acute PID, and the two therapies have a synergistic effect, which can better relieve pain and symptoms. Wu et al. [82] investigated the effect of acupuncture on the inflammation and symptoms of patients suffering from acute PID. The efficacy of observation arm was better than that of control arm $(95.24 \%$ vs $81.08 \%, P<0.05)$. After treatment, the levels of TNF- $\alpha$ and CRP in the serum were significantly decreased $(P<0.05)$. Shi [83] also got a similar conclusion. Liu et al. [84] conducted a multicenter RCT. The results showed that acupuncture combined with ibuprofen sustained-release capsule can effectively improve the symptoms and signs of patients with chronic pelvic pain caused by PID and improve their quality of life, which was more effective than ibuprofen sustained-release capsule alone. Several systematic reviews and meta-analyses of acupuncture treatment of PID have been published successively. Although these studies had varying degrees of bias risk, the conclusions still provided strong evidence for acupuncture as supplements to substitute Western medicine to treat PID. Zheng et al. [85] used data mining technology to analyze the rule of acupoint selection in treating CPID. The results showed that acupoints of 14 meridians were mainly selected for treating CPID, of which CV4 (168 times) and SP6 (155 times) were used most frequently, with the proportion as high as $76.02 \%$ and $70.14 \%$, respectively. According to the analysis of the regularity of the use of extra meridian acupoints, EX-CA1 has the highest frequency followed by EX-B7 and Changyi. He et al. [86] conducted a meta-analysis on the efficacy and safety of acupuncture in treating CPID. The results showed that, in terms of efficacy, the total effective rates of acupuncture and its combination with medicine were higher than that of medicine alone; there was no significant difference in the incidence of adverse reactions in acupuncture arm compared with the control arm. We have listed the 10 most commonly used acupoints for the treatment of PID in Table 3.

3.2. The Mechanism of Acupuncture in Treating PID. Studies have shown that acupuncture induces reactions such as activation of nerve, endocrine, and immune signaling pathways by stimulating skin tissue [87]. There are many pathogenic factors of PID [88], including a variety of microbial infections, decreased autoimmunity, and pelvic floor muscle dysfunction. The main mechanisms of acupuncture in treating PID are as follows: first of all, acupuncture can promote blood circulation, increase the permeability of the cell membrane, and accelerate the absorption of inflammation, thereby treating PID; second, acupuncture can enhance immunity by stimulating health-care acupoints and specific acupoints, thereby preventing the occurrence of PID and promoting the recovery of the disease; and finally, acupuncture treats PID by improving persistent pain in the lower abdomen. Now, we will discuss these three aspects in detail in the following sections.

3.2.1. Acupuncture Could Promote Blood Circulation and Accelerate Inflammation Absorbed. Acupuncture can promote blood circulation, dilate blood vessels and lymphatic vessels, increase the permeability of cell membranes, exhibit an anti-inflammatory effect to a certain extent, and promote 
TABLE 3: The location, regional anatomy, and innervation of common acupoints for treating PID.

\begin{tabular}{|c|c|c|c|}
\hline Acupoint & Location & Muscle & Innervation \\
\hline $\begin{array}{l}\text { CV4 } \\
\text { (Guanyuan) }\end{array}$ & $\begin{array}{l}3 \text { cun below the center of the umbilicus on the lower abdomen and } \\
\text { on the anterior midline }\end{array}$ & Fibrous tissue, linea alba & $\mathrm{L} 1$ \\
\hline $\begin{array}{l}\text { SP6 } \\
\text { (Sanyinjiao) }\end{array}$ & 3 cun proximal to the medial malleolus & $\begin{array}{l}\text { Mm. flexor digitorum longus, tibialis } \\
\text { posterior }\end{array}$ & L4-5, S1-2 \\
\hline $\begin{array}{l}\text { EX-CA1 } \\
\text { (Zigong) }\end{array}$ & 4 cun below the umbilicus and 3 cun lateral to the anterior midline & $\begin{array}{l}\text { Obliquus internus abdominis, } \\
\text { musculus transversus abdominis }\end{array}$ & T10-L2 \\
\hline CV3 (Zhongji) & 4 cun caudal to the umbilicus & Fibrous tissue, linea alba & L1 \\
\hline ST36 (Zusanli) & $\begin{array}{l}3 \text { cun below ST35, one finger breadth from the anterior crest of the } \\
\text { tibia, (front ridge of tibia), between fibula and tibia }\end{array}$ & $\begin{array}{l}\text { Anterior tibial muscle, extensor } \\
\text { digitorum longus }\end{array}$ & L4-5, S1-2 \\
\hline CV6 (Qihai) & $\begin{array}{l}1.5 \text { cun inferior to the center of the umbilicus, on the anterior } \\
\text { midline }\end{array}$ & Fibrous tissue, linea alba & Th11 \\
\hline ST29 (Guilai) & $\begin{array}{c}4 \text { cun inferior to the center of the umbilicus, } 2 \text { cun lateral to the } \\
\text { anterior midline }\end{array}$ & M. rectus abdominis & Th6-12 \\
\hline BL32 (Ciliao) & $\begin{array}{c}\text { At the } 2 \text { nd posterior sacral foramen on the sacrum and the } \\
\text { posterior ramus of the S2 nerve }\end{array}$ & Erector spinae & L2-S4 \\
\hline BL23 (Shenshu) & Under the 2 nd spinous process of lumbar vertebra, next to 1.5 cun & Erector spinae & L1 \\
\hline $\begin{array}{l}\text { SP9 } \\
\text { (Yinlingquan) }\end{array}$ & Below medial tibia condyle & M. gastrocnemius & S1-2 \\
\hline
\end{tabular}

the dissipation and absorption of pathological products and inflammatory exudates [89-91]. Shi [83] randomly divided 70 CPID patients into control arm (conventional therapy) and observation arm (conventional therapy combined with acupuncture). After treatment, the imageology showed that the time of inflammation absorbed and abdominal pain relief in the observation arm were shorter than those of control arm, with a statistically significant difference $(P<0.05)$. The levels of TNF- $\alpha$, hs-CRP, and IL-6 in the two arms were decreased, and the observation arm was lower than the control arm $(P<0.05)$. Therefore, acupuncture can effectively increase the absorption of inflammation, which could be recommended for the treatment of PID [71].

3.2.2. Acupuncture Can Improve Immunity. The body mainly relies on the immune system to fight infection, with innate immunity in the early stage and acquired immunity in the later stage. Under the stimulation of pathogenic bacteria, the body activates its autoimmune system and releases plenty of cytokines and chemokines through a series of signal transduction, thereby killing the pathogenic bacteria. In the reproductive system, the innate immune system, namely Toll-like receptors (TLRs), is the first to be activated [91]. It plays an important role in the host's antimicrobial infection by regulating innate and acquired immunity and is the key to linking infection, inflammation, and injury [92]. Studies have shown that the variation of Toll-like receptors is closely related to the occurrence and development of PID [93]. Huang conducted an RCT on 80 patients with PID, and the conclusion showed that the application of acupuncture combined with acupoint injection in treating patients with CPID can improve patients' immunity, enhance efficacy, and reduce relapse [94]. Research by Jiang Yu et al. pointed out that acupuncture at Dong Qi points (a special acupuncture therapy popular in Taiwan, Europe, and the United States) can enhance the immunity of patients with CPID [95].
3.2.3. Acupuncture Can Relieve Lower Abdominal Pain. Pain located at the lower abdomen and lumbosacrum is the most common symptom of PID, which affects the life quality of most patients [96]. Numerous studies have confirmed that acupuncture can effectively relieve pain [88]. The experiment by Wang explored the efficacy of warming-needle moxibustion in treating CPID with the type of qi stagnation and blood stasis and concluded that warming-needle moxibustion could significantly eliminate pain and other clinical manifestations of CPID patients and relieve their anxiety and depression [97]. Gr [98] explored the efficacy of acupuncture combined with moxibustion in treating CPID. The results showed that, after treatment, the NRS pain score of the treatment arm was lower than that of the control arm $(P<0.05)$, and the total effective rate was higher than that of the control arm $(P<0.05)$.

3.3. The Safety of Acupuncture in Treating PID. Acupuncture is relatively safe on most occasions, only improper operation performed by unqualified acupuncturists can cause adverse reactions such as faint during treatment, stuck needle, bending of the needle, needle breakage, and hematoma. Compared with conventional therapies, treating PID with acupuncture has fewer adverse reactions, most of which are skin erythema, bruising, and pain [99]. These problems usually can be reduced or avoided by careful operation. According to an RCT, the incidence of adverse reactions in the acupuncture arm was $0.75 \%$, which was significantly lower than $25.00 \%$ in the control arm $(P<0.05)$ [84]. In another clinical trial of 62 patients with pain treated by acupuncture, only 5 patients had adverse events [100]. Compared with significant efficacy, most minor adverse reactions can be ignored [101].

At present, a large number of clinical trials have shown that acupuncture has a clear effect on PID, and the mechanism is also relatively clear. However, there are still some limitations such as insufficient sample size and lack of high- 
quality evidence. Therefore, large-scale RCTs are needed to further verify the efficacy and mechanism of acupuncture in treating PID.

3.4. The Efficacy and Mechanism of Moxibustion in Treating $P I D$. As a natural therapy, moxibustion is to fumigate acupoints by igniting moxa-sticks made from artemisia to treat diseases. Its mechanism is similar to acupuncture, and they have a synergistic effect in treating diseases. Moxibustion is commonly used to treat gynecological diseases such as PID, dysmenorrhea, premature ovarian failure, blocked fallopian tubes, and infertility [102-106]. Among different types of moxibustion, heat-sensitive moxibustion and medicine-separated moxibustion are most frequently utilized to treat PID. Heat-sensitive moxibustion, also known as "heat-sensitive suspension moxibustion," refers to using the heat generated by burning moxa-sticks to stimulate heat-sensitive moxibustion sensation (e.g., heat permeability, heat expansion, heat transfer, local heatless but remote heat, nonheat sensation) to promote pelvic blood circulation, accelerate the absorption, and dissipation of inflammation, thereby treating PID [107, 108]. It has the characteristics of without touching the body, injury, side effects, etc. Yin's RCT compared the efficacy of acupuncture and heat-sensitive moxibustion in treating CPID. The results showed that the efficacy of heat-sensitive moxibustion was better than that of acupuncture, and the effective rates were $93.33 \%$ vs $77.78 \%(P<0.05)$ [109]. Wang et al. randomly assigned 208 cases with TCM syndrome type of qi stagnation and blood stasis CPID into Xuefu Zhuyu capsule arm and heat-sensitive moxibustion Ren Du meridian combined with Xuefu Zhuyu capsule arm. The results showed that the effect of heat-sensitive moxibustion on Ren Du meridian combined with Xuefu Zhuyu capsule was better. Meanwhile, it can significantly reduce the levels of CA125 and IL-8 and increase the level of TGF- $\beta 1$ in serum. Therefore, heatsensitive moxibustion plays an important role in treating CPID [110]. Medicine-separated moxibustion is a combination of moxibustion, acupoints, and medicine. Medicineseparated moxibustion is an effective treatment for CPID with TCM syndrome type of cold-damp stagnation, which fully combines the efficacy of TCM with the heat produced by moxibustion. Because the skin around the umbilical cord is thin, it is easier for the drug to reach the lesion directly, thereby improving the arterial blood supply of the uterus, promoting the absorption of pelvic inflammation, and achieving the purpose of treating disease [111]. Some studies have shown that moxibustion can promote metabolism, increase hematopoiesis of erythrocytes, leukocytes, hemoglobin, and enhance immunity $[112,113]$. In addition, studies have confirmed that moxibustion could significantly reduce the levels of CRP, IL-2, IL-6, and TNF- $\alpha$ in the serum of CPID patients and could effectively control the patient's infection status $[114,115]$. At present, there are few adverse reactions related to moxibustion in treating PID. The adverse reactions could be that the burning moxa-sticks fall off then scald the skin and the symptoms such as fever, thirst, and skin pruritus after moxibustion. In short, moxibustion has its unique advantages in treating PID. However, there still lacks research on moxibustion's heat-sensitive effect and thermal radiation effect, which should be the focus of future research.

\section{The Overview of Other CAMs in Treating PID}

In addition to acupuncture and CHM, pelvic exercises, hyperbaric oxygen therapy, and other therapies can also treat PID.

4.1. The Application of Pelvic Exercises in Treating PID. Pelvic exercise belongs to aerobic exercise, which can increase the tension of pelvic ligaments and blood vessels, improve pelvic blood circulation, and promote inflammatory absorption [116]. In addition, pelvic exercise can enhance the pelvic floor ligament and muscle strength, promote local oxygen uptake, and relieve symptoms such as lumbosacral pain and lower abdominal distension $[117,118]$. Pelvic exercise, the behavioral therapy in psychotherapy, can improve symptoms, at the same time, it can also help patients adjust their mentality in time, establish the confidence to cure the disease, ensure patient compliance with treatment, and improve clinical efficacy [119]. A large number of studies have found that pelvic exercise can effectively reduce the level of immune factors, such as TNF- $\alpha$, CRP, IL-2, IL-6, IL-10, and MDA, and help to relieve abdominal pain. It is safe and worthy of clinical application [120-123].

\subsection{The Application of Hyperbaric Oxygen Therapy in Treating} $P I D$. Hyperbaric oxygen therapy has the function of increasing blood oxygen content and oxygen partial pressure and improving the state of systemic organs, which can be used to treat gynecological diseases such as infertility, premature ovarian failure, and PID [124-126]. Studies have shown that hyperbaric oxygen has a bactericidal effect, especially on anaerobic bacteria, and can inhibit the growth of aerobic bacteria $[127,128]$. Other studies have shown that hyperbaric oxygen plays a role in the treatment of PID by downregulating CRP and inflammatory cytokines [129]. Hyperbaric oxygen can also improve the body's immunity and reduce the recurrence and complications of PID [130]. Many pieces of literature reported that hyperbaric oxygen combined with the drug could increase its effect, which was worthy of further clinical application $[131,132]$.

\subsection{The Application of Microwave Physiotherapy in Treating} PID. Microwave physiotherapy, complementary therapy for PID, can dilate blood vessels, accelerate blood circulation and metabolism, and improve tissue nutrition. A large number of experiments have confirmed that microwave physiotherapy combined with TCMRE can accelerate the absorption of pathological and inflammatory products, promote local blood circulation, and significantly improve inflammatory factors and hemorheological indicators [133-136]. Microwave physiotherapy can also improve the 
patients' ability to prevent disease and effectively prevent the recurrence of PID [137, 138]. Some RCTs have shown that mild moxibustion combined with microwave physiotherapy can promote blood circulation, accelerate metabolism, and effectively alleviate the symptoms of CPID patients with qi stagnation and blood stasis [139-141].

4.4. The Application of Cupping in Treating PID. Cupping is an external therapy of TCM to prevent and treat diseases. A negative pressure environment is formed in the cupping through methods such as combustion and suction. After cupping is adsorbed on acupoints or corresponding parts of the body, the local tissue would be congested, thereby achieving the purpose of warming the channels and unblocking the collaterals, relieving swelling and pain, drawing out the toxin, and expelling pus [142]. Modern studies have shown that cupping can regulate the function of the nervous system, improve the function of phagocytes, and promote blood circulation. Therefore, it has been proven to be one of the unique ways to treat PID [143]. The negative pressure stimulation produced by cupping can not only promote pelvic blood circulation, increase metabolism, be beneficial to the absorption of inflammation, and repair damaged tissues but also enhance immunity, shorten the course of treatment, and reduce the recurrence rate [144-146].

4.5. The Application of Ozone Therapy in Treating PID. Ozone is a highly active molecule with antioxidant activity, which can be used as a complementary alternative therapy for PID [147-149]. Through animal comparative studies, Wei et al. found that $\mathrm{O} 3$ can treat PID by inhibiting the necrosis of endometrial epithelial cells and reducing the inflammatory response, which provides a new target for the treatment of PID [150]. Escandón et al. [151] researched that ozone can reduce endometritis and improve the fertility of dairy cows. Ozone therapy is becoming a new adjuvant therapy for female reproductive health.

\section{Summary}

In recent years, the incidence of PID has reached as high as $12 \%$, and the risk of depression secondary to PID has also increased yearly [152]. Clinical treatment is mainly focusing on antibiotics. However, antibiotic abuse is severe, and the potential risks such as flora imbalance, bacterial resistance, super bacteria production, and increased adverse reactions have become increasingly prominent. The most commonly used CAMs for treating PID are acupuncture and CHM. In addition, this review also mentioned the application of pelvic exercises, hyperbaric oxygen therapy, microwave physiotherapy, cupping, and ozone therapy in PID. In summary, the merits of CAM in the treatment of PID mainly include the following: (1) CAM effectively alleviates the symptoms caused by PID and accelerates the disappearance time of symptoms; (2) CAM can greatly improve clinical effective rate and reduce adverse reactions and recurrence rates; and (3) the efficacy produced by CAM can replace antibiotics and reduce the dose of antibiotics required for PID treatment. As an adjuvant treatment of PID, CAM's controversial efficacy and mechanism have aroused the horizon of many Western medical scholars. At present, due to the limitations such as small sample size, low quality, and lack of uniform standards, the effectiveness of CAM in the treatment of PID has been controversial. Therefore, we are looking forward to more high-quality research on CAM in the treatment of PID to provide a more convincing basis for CAM treatment of PID.

\section{Data Availability}

No data were used to support this study.

\section{Conflicts of Interest}

The authors declare that there are no conflicts of interest.

\section{Authors' Contributions}

Dongmei Wang, Yue Jiang, and Jiaxing Feng contributed equally to this work.

\section{Acknowledgments}

This work was supported by the Natural Science Foundation of Heilongjiang (grant no. H2017051), the Scientific Research Project of Heilongjiang Administration of Chinese Medicine (grant no. SHY18-068), and the General Project of Heilongjiang Provincial Department of Education (grant no. SJGY20170613).

\section{References}

[1] C. Wang, J. Chen, Y. Xiao, and Q. Shen, "Guizhi Fuling wan for chronic pelvic inflammatory disease protocol," Medicine, vol. 99, no. 51, p. e23549, 2020.

[2] A. Curry, T. Williams, and M. L. Penny, "Pelvic inflammatory disease: diagnosis, management, and prevention," American Family Physician, vol. 6, no. 100, pp. 357-364, 2019.

[3] R. F. Savaris, D. G. Fuhrich, J. Maissiat, R. V. Duarte, and J. Ross, "Antibiotic therapy for pelvic inflammatory disease," Cochrane Database of Systematic Reviews, vol. 8, p. CD010285, 2020.

[4] R. C. Brunham, S. L. Gottlieb, and J. Paavonen, "Pelvic inflammatory disease," New England Journal of Medicine, vol. 372, no. 21, pp. 2039-2048, 2015.

[5] W. L. Qin, "Efficacy observation of retention enema of pelvic inflammatory soup combined with low dose fleroxacin in the treatment of chronic pelvic inflammatory disease," Shaanxi Journal of Traditional Chinese Medicine, vol. 36, pp. 263-264, 2015.

[6] L. D. Han and L. Liu, "Reproductive health survey about women at childbearing age in Changsha," Journal of Reproductive Medicine, vol. 24, pp. 46-50, 2015.

[7] L. H. Jiang and H. Hao, "Epidemiological characteristics of female reproductive tract infections and effects of hyperbaric oxygen therapy," Chinese J. Nosocomiology, vol. 25, pp. 931-933, 2015. 
[8] P. T. Jiang, "Survey and analysis of women's diseases in primary hospital," Forest Industries, vol. 9, no. 13, p. 112, 2012.

[9] C. W. Bugg, T. Taira, and M. Zaurova, "Pelvic inflammatory disease," Obstetrics \& Gynecology, vol. 116, no. 2, pp. 419428, 2010.

[10] S. Katarzyna, M.-B. Beata, and E. Rudnicka, "The impact of selected bacterial sexually transmitted diseases on pregnancy and female fertility," International Journal of Molecular Sciences, vol. 22, no. 4, 2021.

[11] M. Safrai, A. Rottenstreich, and S. Asher, "Risk factors for recurrent pelvic inflammatory disease," European Journal of Obstetrics \& Gynecology and Reproductive Biology, vol. 244, 2020.

[12] C. W. Bugg and T. Taira, "Pelvic inflammatory disease: diagnosis and treatment in the emergency department," Emergency Medicine Practice, vol. 12, no. 18, 2016.

[13] W. L. Risser, J. M. Risser, and A. L. Risser, "Current perspectives in the USA on the diagnosis and treatment of pelvic inflammatory disease in adolescents," Adolescent Health, Medicine and Therapeutics, vol. 8, pp. 87-94, 2017.

[14] W. Ka, "Centers for disease control and prevention sexually transmitted diseases treatment guidelines," Clinical Infectious Diseases, vol. 61, no. 8, 2015.

[15] L. Chen, Y. Zhang, and K. Sai, "Applications and therapeutic actions of complementary and alternative medicine for women with genital infection," Evidence-Based Complementary and Alternative Medicine, vol. 2014, 2014.

[16] M. Bahall and G. Legall, "Knowledge, attitudes, and practices among health care providers regarding complementary and alternative medicine in Trinidad and Tobago," BMC Complementary and Alternative Medicine, vol. 17, no. 1, p. 144, 2017.

[17] C. Yu, S. Wei, and H. Li, "Fuke qianjin combined with antibiotic therapy for pelvic inflammatory disease: a systematic review and meta-analysis," Evidence-Based Complementary and Alternative Medicine, vol. 2020, Article ID 5372839, 10 pages, 2020.

[18] W. Wang, Z. Hua, and W. Wang, "Current policies and measures on the development of traditional Chinese medicine in China," Pharmacological Research, vol. 163, 2021.

[19] J.-F. Xia, Y. Inagaki, J.-F. Zhang, L. Wang, and P.-P. Song, "Chinese medicine as complementary therapy for female infertility," Chinese Journal of Integrative Medicine, vol. 23, no. 4, pp. 245-252, Apr. 2017.

[20] W. Rf, "Efficacy of Kangfu anti-inflammatory suppositurecombined with antibiotics in the treatment of pelvic inflammation," Chinese Journal of Clinical Ration Drug Use, vol. 6, pp. 98-99, 2017.

[21] Y. M. Zhang and L. R. Zhang, "Clinical effect of dachaihu decoction on acute pelvic inflammation and its effect on secretory immunoglobulin A and serum CRP," Hebei Medical University, vol. 27, pp. 326-330, 2021.

[22] Y. L. Xiangli, "Clinical observation of Gongyingtuling decoction combined with moxifloxacin in the treatment of pelvic inflammatory disease," Guangming Journal of New Chinese Medicine, vol. 35, pp. 3628-3630, 2020.

[23] J. L. Feng, "The value of Hongteng miso decoction in the treatment of chronic pelvic inflammation," Mod. Med. Heal. Res. Electron. J.vol. 3, pp. 93-94, 2019.

[24] L. J. Wang and Q. X. Gan, "Effect of modified Yiqi Huayu Penyan decoction on treatment of pelvic inflammation of Qixu Xueyu syndrome and its influence on blood microcirculation," Shaanxi Journal of Traditional Chinese Medicine, vol. 41, pp. 1722-1726, 2020.

[25] L. Z. Xiong, Q. Y. Wang, and X. X. Zeng, "Effects of Dahuang Mudanpi decoction on immune status of sequelae of pelvic inflammatory diseases," Chongqing Medicine, vol. 47, pp. 3467-3470, 2018.

[26] Y. H. Xiong, "Clinical observation on Qingre huayu decoction in the treatment of pelvic inflammatory diseases," Chinese Medicine Modern Distance Education of China, vol. 17, pp. 41-43, 2019.

[27] X. L. Feng, J. Sha, and C. Jing, "Effect of Fuyanshu Capsules combined with antibiotics on inflammatory factors in patients with pelvic inflammatory disease," Zhongguo Zhongyao Zazhi, vol. 44, no. 12, pp. 2637-2643, 2019.

[28] Z. L. Ma J, L. Ding, and Y. Wang, "Therapeutic effects of danbai granules combined with azithromycin on chronic pelvic inflammatory disease," World Chinese Med.vol. 15, pp. 2430-2433, 2020.

[29] G. H. Zhang, "Clinical effect observation of Fuyaning decoction combined with antibiotics in the treatment of chronic pelvic inflammation," Practical Clinical Journal of Integrated Traditional Chinese and Western Medicine, vol. 20, pp. 71-72, 2020.

[30] W. Hb, "Curative effect of guizhi fuling pills on chronic pelvic inflammatory disease and its influence on hemorheology, oxidative stress and levels of serum hs-CRP, TGF$\beta 1$, GM-CSF and complement C3," Chinese Arch. traditional Chinese medicine, vol. 38, pp. 214-217, 2020.

[31] Y. Yang, Y. Lu, and D. Zhou, "Effectiveness of Jinying capsule on pelvic inflammatory disease in patients with symptom of damp and heat accumulation: a double-blinded, multicenter, randomized, placebo-controlled clinical trial," Journal of Traditional Chinese Medicine, vol. 36, no. 2, pp. 163-167, 2020.

[32] W. M. Zhou and M. Y. Chen, "Treatment of chronic endometritis by penning granule: a clinical randomized controlled trial," Zhongguo Zhong Xi Yi Jie He Za Zhi, vol. 36, pp. 1055-1060, 2016.

[33] L. Q. Wu, Y. P. Fu, and S. S. Lv, "Clinical observation of Tongluo Qingre decoction on sequelae of pelvic inflammatory disease with stagnation of qi, blood stasis and stagnation of heat type," Journal of Practial Traditional Chinese Medicine, vol. 37, pp. 199-200, 2021.

[34] Q. L. Huang, "Effect of pelvic inflammatory decoction in treatments of chronic pelvic inflammatory diseas," Acta Chinese Medicine, vol. 31, pp. 734-737, 2016.

[35] Z. N. Lan, L. L. Zhang, and H. Liu, "Effect of shaofu Zhuyu decoction on chronic pelvic inflammation with cold coagulation and blood stasis," Medical Journal of Chinese People's Liberation, vol. 33, pp. 92-94, 2021.

[36] L.-J. Zhang, J.-Y. Zhu, M.-Y. Sun et al., “Anti-inflammatory effect of Man-Pen-Fang, a Chinese herbal compound, on chronic pelvic inflammation in rats," Journal of Ethnopharmacology, vol. 208, pp. 57-65, 2017.

[37] W. Zou, L. Gong, F. Zhou et al., "Anti-inflammatory effect of traditional Chinese medicine preparation Penyanling on pelvic inflammatory disease," Journal of Ethnopharmacology, vol. 266, p. 113405, 2021.

[38] Y. Zheng, Y. Jin, H. B. Zhu, S. T Xu, Y. X Xia, and Y Huang, "The anti-inflammatory and anti-nociceptive activities of Patrinia villosa and its mechanism on the proinflammatory cytokines of rats with pelvic inflammation," African Journal of Traditional, Complementary, and Alternative Medicines: AJTCAM, vol. 9, no. 3, pp. 295-302, 2012. 
[39] S. Z. Sun, "Clinical efficacy of Fuke Qianjin Tablet combined with Levofloxacin in the treatment of chronic pelvic inflammatory disease and its effect on inflammatory factors," Chinese Journal of Clinical Rational Drug Use, vol. 13, pp. 122-124, 2020.

[40] B.-H. Xia, L. Bai, and Y.-S. Zhang, "Mechanisms of Fuke Qianjin Capsules in treatment of pelvic inflammatory disease based on GC-MS metabolomics," Zhongguo Zhongyao Zazhi, vol. 44, no. 22, pp. 4940-4946, 2019.

[41] L. Xiao, Y. R. Liu, and J. D. Hai, "Research on the mechanism of Chinese herbal medicine Radix Paeoniae Rubra in improving chronic pelvic inflammation disease by regulating PTGS2 in the arachidonic acid pathway," Biomedicine \& Pharmacotherapy, vol. 129, 2020.

[42] L. X. Jiang and J. L. He, "Study on core Chinese herbal medicines and mechanism in Chinese herbal medicine enema for treatment of chronic pelvic inflammation by data mining and network Pharmacology," Journal of Guangzhou University of Traditional Chinese, vol. 38, pp. 379-385, 2021.

[43] H. Tilg, N. Zmora, T. E. Adolph, and E. Elinav, "The intestinal microbiota fuelling metabolic inflammation," Nature Reviews Immunology, vol. 20, no. 1, pp. 40-54, 2020.

[44] L. M. Feng, Y. G. Han, F. Y. Sun, G. Chen, and K. L. Xu, "Progress in clinical research and application of traditional Chinese medicine enema," World Journal of Integrated Traditional and Western Medicine, vol. 15, pp. 1565-1568, 2020.

[45] L. S. Feng, "Effect of traditional Chinese medicine retention enema on chronic pelvic inflammatory disease," Inn. Mong. J. Tradit. Chinese Med, vol. 33, no. 27, pp. 88-89, 2014.

[46] W. Hb and F. Jw, "Progress of traditional Chinese medicine retention enema in the treatment of chronic pelvic inflammatory disease," Journal of Sichuan Traditional Chinese Medicine, vol. 30, pp. 56-58, 2012.

[47] L. Ma, Y. Q. Zhang, G. M. Li, and C. J. Gao, "Curative effect of traditional Chinese medicine enema on chronic pelvic inflammatory disease and its effect on indexes of hemorheology, inflammation and oxidative stress," Modern Journal of Integrated Traditional Chinese and Western Medicine, vol. 28, pp. 822-825, 2019.

[48] S. Kong, Z. Yue, and L. Chen, "The complementary and alternative medicine for endometriosis: a review of utilization and mechanism," Evidence-Based Complementary and Alternative Medicine, vol. 2014, 2014.

[49] B. Jia, "Clinical effect of comprehensive nursing intervention combined with Kangfuyan capsule retention enema in the treatment of pelvic inflammatory disease," China Practical Medicine.vol. 36, no. 2, pp. 304-306, 2021.

[50] R. Rf, "Application effect of retention enema combined with holistic nursing in patients with chronic pelvic inflammatory disease," Guangming Chinese Medical Journal, vol. 36, pp. 304-306, 2021.

[51] Z. Liang and N. Ling, "Clinical observation of Chinese medicine collapse stains combined with Chinese herbal retention enema for sequelae of pelvic inflammatory disease," Journal of Liaoning University of Traditional Chinese Medicine, vol. 21, pp. 38-40, 2019.

[52] L. J. Li and J. F. Fu, "The effect of retention enema of traditional Chinese medicine on chronic pelvic inflammatory disease and inflammatory factors," World Chinese Medine, vol. 12, pp. 772-775+779, 2017

[53] X. Y. Shao, "The curative effect and experience of traditional Chinese medicine enema in the treatment of pelvic inflammatory diseases," Guangming Journal of Chinese Medicine, vol. 35, pp. 2165-2167, 2020.

[54] M. Li, "Study on the curative effect of oral and retention enema of No. 1 decoction of gynecology on chronic pelvic inflammation," Journal of China Prescr. Drug, vol. 17, pp. 106-107, 2019.

[55] X. L. Xie, "To observe the pelvic inflammation orally and the clinical curative effect of retention enema treatment of chronic pelvic inflammatory disease," Sichuan Medical Journal, vol. 36, no. 12, pp. 1680-1682, 2015.

[56] S. Wang, "Clinical efficacy of xiaoyan decoction combined with retention enema in the treatment of chronic pelvic inflammation," Mediterranean Journal of Mathematics, vol. 33, pp. 1810-1812, 2020.

[57] Y. H. Yao, "Clinical study on penqiangyan prescription combined with Chinese herbal retention enema for patients with oviduct obstructive infertility after hysteroscopic surgery," Chinese Medical Journal, vol. 51, pp. 204-209, 2019.

[58] C. Ling and L. L. Luo, "Observation on the curative effect of Fuyan decoction retention enema for tubal obstructive infertility," Journal Practical Traditional Chinese Medicine, vol. 37, no. 2, pp. 268-269, 2021.

[59] F. Y. L. T. T. Zhai, "Clinical observation of 25 cases of infertility caused by chronic pelvic inflammatory disease treated with enema supplemented by Professor Ding Lixian's experience formula "Penyanqing" after laparoscopic surgery," Shanxi University of Traditional Chinese Medicine, vol. 21, pp. 143-145, 2020.

[60] M. W. Dong L, H. Z. Miao, and D. Qu, "Clinical study on treatment of tubal infertility after laparoscopic operation combined with traditional Chinese medicine enema," Qiqihar Hospital Traditional Chinese Medicine, vol. 2021, 2021.

[61] X. H. Wang, "Effect observation of antibiotic combined with retention enema of traditional Chinese medicine for chronic pelvic inflammatory," Chinese Journal of Clinical Rational Drug Use, vol. 10, pp. 48-49, 2017.

[62] Q. D. Mao, "Effect and recurrence rate of traditional Chinese medicine retention enema in the treatment of pelvic inflammatory disease," Infection and Immunity Ed.vol. 24, pp. 122-123, 2020.

[63] L. Hu, C. Yuqi, and T. Chen, "A systematic study of mechanism of sargentodoxa cuneata and Patrinia scabiosifolia against pelvic inflammatory disease with dampnessheat stasis syndrome via network Pharmacology approach," Frontiers in Pharmacology, vol. 11, 2020.

[64] Y. Zhang, X. Pan, S. Ran, and K. Wang, "Purification, structural elucidation and anti-inflammatory activity in vitro of polysaccharides from Smilax China L," International Journal of Biological Macromolecules, vol. 139, pp. 233-243, 2019.

[65] D. Kong, P. Fu, Q. Zhang, X. Ma, and P. Jiang, "Protective effects of Asiatic acid against pelvic inflammatory disease in rats," Experimental and Therapeutic Medicine, vol. 17, no. 6, pp. 4687-4692, 2019.

[66] Y. Zhuang, J.-j. Xing, J. Li, B.-Y. Zeng, and F.-r. Liang, "History of acupuncture research," International Review of Neurobiology, vol. 111, pp. 1-23, 2013.

[67] K. Tj, "Acupuncture: theory, efficacy, and practice," Annals of Internal Medicine, vol. 136, no. 5, pp. 374-383, 2002.

[68] M. Mj and B. Se, "Puncturing the myths of acupuncture," Journal of Integrative Medicine, vol. 14, no. 5, pp. 311-314, 2016. 
[69] J.-C. Shen, X. Rui, A. S. S. Lynn, and Z.-B. Yang, "Analysis of the development status of acupuncture and moxibustion in Malaysia," Zhongguo Zhen Jiu, vol. 39, no. 8, pp. 871-873, 2019.

[70] Y. S. Wu and D. J. Cai, "“Current situation and prospect of acupoint efficacy research," Traditional Chinese Medicine and Clinical Pharmacology, vol. 35, pp. 4785-4787, 2020.

[71] H. N. Yin, J. N. Li, and Q. Li, "Development, inheritance and innovation of Chinese acupuncture," Traditional Chinese Medicine and Clinical Pharmacology, vol. 34, pp. 4467-4470, 2019.

[72] Y. Y. Yang, Z. X. Shen, and Z. C. Wu, "Strategic planning of the "belt and road" development of acupuncture and moxibustion," Chinese Acupunct Moxibustion, vol. 37, pp. 343-348, 2017.

[73] L. L. Yang Yy, "Chinese acupuncture of the world federation of acupuncture societies has entered the countries along the 'one belt and one road' route," Shanghai Cooperation Organisation, vol. 22, pp. 45-46, 2017.

[74] J. S. Han, "Study on analgesia of needle anaesthesia," Acupuncture Research, vol. 41, pp. 377-387, 2016.

[75] H. N. Chen Yb, F. Fang, and Y. X. Ma, "Research on the development and characteristics of European acupuncture before the 20th century," Journal of Clinical Acupuncture and Moxibustion, vol. 36, pp. 74-78, 2020.

[76] Y. Chen, Y. Yuan, and Y Jin, "Acupuncture for chronic pelvic inflammatory disease: a systematic review protocol," Medicine (Baltimore), vol. 97, no. 13, 2018.

[77] B. Kc, K. Ja, and D.-K. S, "Acupuncture in obstetrics and gynecology," Obstetrical and Gynecological Survey, vol. 74, no. 4, 2019.

[78] H. Chen and C. E. D. Lim, "The efficacy of using acupuncture in managing polycystic ovarian syndrome," Current Opinion in Obstetrics and Gynecology, vol. 31, no. 6, pp. 428-432, 2019.

[79] X. M. Wang, "On the therapeutic efficacy of electric acupuncture with moxibustion in 95 cases of chronic pelvic infectious disease (PID)," Journal of Traditional Chinese Medicine, vol. 1989, 1989.

[80] L. X. Zhen and Y. Wang, "Observation on therapeutic effect of warming needle moxibustion on chronic pelvic inflammation of cold-damp stagnation type," Chinese Acupuncture \& Moxibustion, vol. 28, pp. 736-738, 2008.

[81] L. L. Zhang, "Clinical effect of acupuncture combined with traditional Chinese medicine enema on patients with acute pelvic inflammatory disease," Medical Equipment, vol. 33, pp. 126-127, 2020.

[82] . D. Wu, G. Ly, and L. Ps, “"'[Effect of acupuncture on inflammatory cytokines in patients with acute pelvic inflammatory disease]," zhen ci yan jiu=acupunct," Res: Anthropology and Aesthetics, vol. 43, no. 1, 2018.

[83] R. Shi, "Study on the effect of acupuncture and moxibustion in treating chronic pelvic inflammatory disease and its effect on symptom improvement," Chinese Journal of Modern Drug Application, vol. 15, pp. 229-231, 2021.

[84] Y.-H. Liu, X. Wang, and Z. Liang, "Acupuncture combined with western medication on chronic pelvic pain after pelvic inflammatory disease: a multi-center randomized controlled trial," Zhongguo Zhen Jiu, vol. 41, no. 1, pp. 31-35, 2021.

[85] C. Q. Zheng, D. Zhu, Y. Liu, and Yi Liang, "Analysis on the rule of acupoint selection in the treatment of chronic pelvic inflammatory disease by using data mining technology," Shanghai J. Acupunct. Moxibustion, vol. 37, pp. 1214-1219, 2018.
[86] M. Y. He, H. Chen, J. Cheng, Y. B. Xia, and Y. L. Gao, "Metaanalysis of the effectiveness of acupuncture and moxibustion in the treatment of chronic pelvic inflammatory disease," Liaoning Journal of Traditional Chinese Medical Sciences, vol. 46, pp. 1573-1578, 2019.

[87] N. C. Li, M. Y. Li, B. Chen, and Y. Guo, "A new perspective of acupuncture: the interaction among three networks leads to neutralization," Evidence-Based Complementary and Alternative Medicine: eCAM, vol. 2019, p. 2326867, 2019.

[88] T. Peng, Y. Wu, L. Huang, B. He, and S. Wei, "Acupuncture for chronic pelvic pain in patients with SPID," Medicine, vol. 100, no. 4, p. e23916, 2021.

[89] M. B. Sun Kf, M. R. He, Y. F. Ou, X. X. Li, C. F. Zhang, and D. M. Kong, "MA Baozhang's experience in the treatment of tubal inflammatory infertility," Acta Traditional Chinese Medicine and Clinical Pharmacology, vol. 49, pp. 49-53, 2021.

[90] F. Liao, "Effect of acupuncture and moxibustion combined with Yi Guan exercise on hemorheology and inflammatory factors in chronic pelvic inflammation," Guangming Journal of Chinese Medicine, vol. 34, pp. 174-176, 2019.

[91] Q. X. Liu Jj, L. Lu, X. H. Chen, and D. Y. Wang, "Advances in immunological pathogenesis of pelvic inflammatory diseases," Progres en Obstetrique et Gynecologie, vol. 23, pp. 64-66, 2014.

[92] S. C. Liu, "Toll-like receptors and genital tract infection," International Journal of STD \& AIDS, vol. 21, no. 3, pp. 153-157, 2010

[93] T. Bd, F. Re, and N. Rb, "Racial variation in toll-like receptor variants among women with pelvic inflammatory disease," The Journal of Infectious Diseases, vol. 207, no. 6, pp. 940-946, 2013.

[94] X. L. Huang, "Practice study of acupuncture combined with acupoint injection in the treatment of chronic pelvic inflammation," Chinese Journal of Modern Drug Application, vol. 12, pp. 197-199, 2018.

[95] L. S. Jiang, X. S. Zhang, Z. Y. Gong, and X. Q. Zhao, "Effect of acupuncture at Dongshiqi point on immune function in patients with chronic pelvic inflammatory disease," Clinical Journal of Traditional Chinese Medical Sciences, vol. 27, pp. 61-62, 2015

[96] L. S. Yin Bn, "Diagnosis, treatment and evaluation of chronic pelvic pain in gynecology," Chinese Community Dr.vol. 30, pp. 12-14, 2014.

[97] W. X. Wang, "Treatment of chronic pelvic pain after pelvic inflammatory disease with qi stagnation and blood stasis by warm acupuncture," Jilin Journal of Chinese Medicine, vol. 40 , pp. 1380-1382, 2020.

[98] G. R. Shi, "Effect of acupuncture combined with warm acupuncture on pelvic pain after pelvic inflammation," Journal of Practical Traditinal Chinese Medicine, vol. 35, no. 1, pp. 110-111, 2019.

[99] J. Park, Y. Sohn, and A. White, "The safety of acupuncture during pregnancy: a systematic review," Acupuncture in Medicine, vol. 32, no. 3, pp. 257-266, 2014.

[100] P. Moa, S. Liu, and J. Xue, "Clinical research on the comprehensive curative effect of acupuncture and traditional Chinese medicine for pelvic inflammatory sequelae," Medical Science Monitor, vol. 24, 2018.

[101] W. Claudia, D. Pach, and B. Benno, "Safety of acupuncture: results of a prospective observational study with 229,230 patients and introduction of a medical information and consent form," Forsch. Komplementmed.vol. 16, no. 2, pp. 91-97, 2009. 
[102] C.-Y. Kwon, B. Lee, and K. S. Park, "Oriental herbal medicine and moxibustion for polycystic ovary syndrome," Medicine, vol. 97, no. 43, p. e12942, 2018.

[103] D. S. Jiang, Y. C. Zhang, and X. L. Wu, "Observation on curative effect of Ginger-separated Moxibustion at Baliao acupoint combined with Bushen Huoxue Recipe on ovarian reserve function decline," Chinese Acupuncture \& Moxibustion, vol. 37, pp. 1057-1060, 2017.

[104] T. X. Lu and R. T. Liu, "Thermosensitive moxibustion combined with Huoxue Quyu prescription in the treatment of 50 cases of fallopian tube infertility," Jiangxi Journal of Traditinal Chinese Medicine, vol. 47, pp. 67-69, 2016.

[105] L. Q. Ge, "Effect of thermosensitive moxibustion combined with acupoint injection on pregnancy outcome after laparoscopy in infertility patients with tubal fluid accumulation.Journal of Integrated Traditional Chinese and Western Medicine," Modern Journal of Integrated Traditional Chinese and Western Medicine, vol. 27, pp. 1543-1546, 2018.

[106] C. X. Cheng and T. Nie, "Effects of acupuncture combined with moxibustion on inflammatory response indexes and TCM syndrome scores in patients with chronic pelvic inflammatory disease," World Chinese Medicine, vol. 15, pp. 1351-1354, 2020.

[107] H. L. Chen Zy and X. L. Huang, "Clinical application of thermal moxibustion in gynecological diseases," Hunan Journal of Traditinal Chinese Medicine, vol. 36, pp. 179-181, 2020.

[108] P. Z. Ying, "Thermosensitive moxibustion in the treatment of 25 cases of chronic pelvic inflammation," Chinese Medicine Modern Distance Education of China, vol. 15, pp. 123-124, 2017.

[109] Q. Z. Yin, “A randomized parallel control study of acupuncture combined with thermosensitive moxibustion in the treatment of chronic pelvic inflammatory disease (dysmenorrhea/lower belt)," Journal of Practical Traditional Chinese Internal Medicine, vol. 32, no. 5, pp. 62-65, 2018.

[110] W. D. Wang, C. C. Yan, and F. Liu, "Effect of thermal sensitive moxibustion combined with Xuefu Zhuyu capsule on chronic pelvic inflammatory disease with qi stagnation and blood stasis and its effect on serum CA125, IL-8 and TGF- $\beta 1$," Shanghai Journal of Acupuncture Moxibustion, vol. 38, pp. 389-393, 2019.

[111] K. F. Li and Y. F. Chai, "Clinical observation of different duration Chinese medicine umbilical moxibustion in the treatment of chronic pelvic inflammation with cold and dampness stagnation," Clinical Journal of Chinese Medicine, vol. 12, pp. 90-93, 2020.

[112] L. Lan, X. R. Chang, and J. Shi, "Advances of the mechanism of moxibustion," Chinese Arch. Traditinal Chinese Medicine, vol. 29, pp. 2616-2620, 2011.

[113] C. B. Yu, X. M. Luo, and M. L. Zhang, "Clinical study on the treatment of chronic pelvic inflammatory disease by moxibustion combined with traditional Chinese medicine," Clinical Journal of Chinese Medicine, vol. 10, pp. 108-109, 2018.

[114] C. W. Nong, "Effect of moxibustion combined with ligustrazine on chronic pelvic inflammatory disease with cold and dampess and its effect on CRP, IL-6 and TNF- $\alpha$," Journal of Basic Chinese Medicine, vol. 23, pp. 387-389, 2017.

[115] W. Y. Liu and C. M. Yang, "Effect of moxibustion Dong shiqi point combined with microwave on chronic pelvic inflammation and effect on serum IL-2 and TNF- $\alpha$," Shanghai Journal of Acupuncture Moxibustion, vol. 37, pp. 1156-1159, 2018.
[116] Z. F. Chang, "Effect of pelvic health exercise combined with TCM syndrome differentiation in patients with pelvic inflammatory diseases," Journal of Practical Traditional Chinese Internal Medicine, vol. 36, pp. 816-817, 2020.

[117] R. P. Li, "Observation and nursing care of chronic pelvic inflammatory disease treated with traditional Chinese medicine combined with exercise therapy," Journal of Practical Traditional Chinese Internal Medicine, vol. 28, pp. 96-97, 2012.

[118] X. L. Li and Y. Q. Ma, "Clinical observation on the effect of traditional Chinese medicine enema combined with antibiotics and health exercise in the treatment of chronic pelvis inflammation," J. Mod. Integr. Tradit. West. Med.vol. 25, pp. 867-869, 2016.

[119] S. Y. Z. J. F. Gl, "Impact of pelvic muscle exercise and psychological intervention on negative emotions of patients with infertility caused by pelvic inflammatory diseases," Journal of Nursing Sciences, vol. 28, pp. 90-91, 2013.

[120] X. M. Zhou, "Clinical efficacy and serum IL-2, IL-10, TNF- $\alpha$ of patients with chronic pelvic inflammatory disease treated by Puling Penyankang granule combined with pelvic exercise," Inn. Mong. Traditional Chinese Medicine, vol. 36, pp. 40-41, 2017.

[121] W. G. Wang, "Efficacy and safety of Ornidazole combined with pelvic exercises in the treatment of chronic pelvic inflammatory disease," Chinese Journal of Hum. Sex, vol. 28, pp. 108-110, 2019.

[122] L. Y. U. Yan, "Clinical effect of Xiaoyao Pills on the SOD,MDA and interleukin of pa-tients pelvic inflammatory disease," China Mod. Medicine, vol. 24, pp. 73-75, 2017.

[123] F. Q. L. Hong-Yu, "Effect of palmatine sequential combination of exercise therapy on serum inflammatory markers in pelvic inflammation and its clinical effect," Chinese Journal of Biochemical Pharmaceutics, vol. 36, pp. 164-166, 2016.

[124] P. Jesus, O. Carlos, and M. G. Jeasus, "Improvement in serum anti-müllerian hormone levels in infertile patients after hyperbaric oxygen (preliminary results)," JBRA Assisted Reproduction, vol. 19, no. 2, pp. 87-90, 2015.

[125] J. Y. Liu, "Therapeutic effect of hyperbaric oxygen adjuvant therapy on 29 cases of premature ovarian failure," Chinese Journal of Naut. Med High Press Medicine, vol. 27, pp. 510-512, 2020.

[126] Q. Ren, "Hyperbaric oxygen therapy for vulvar dystrophy," Shanxi Journal of Medcine, vol. 39, pp. 334-335, 2010.

[127] Q. L. Lian, "Thinking about medical education of hyperbaric oxygen," Chinese Journal of Naut. Medicine Hyperb. Med, vol. 320 , no. 5, 2008.

[128] F. H. Wang, "Clinical observation of hyperbaric oxygen combined with traditional Chinese medicine in the treatment of chronic pelvic inflammatory disease," Journal of Nursing PLA, vol. 2008, pp. 47-48, 2008.

[129] W. G. Lou Hp, L. L. Ai, and Y. T. Lin, "Clinical efficacy and mechanism of hyperbaric oxygen combined with drugs in the treatment of chronic pelvic inflammation," Chinese Journal of Naut. Med High Press Medicine, vol. 23, pp. 312-314, 2016.

[130] S. G. Li Gh, "Effect of hyperbaric oxygen on treatment of chronic pelvic inflammation in 56 cases," Chinese Journal of Naut. Med High Press Medicine, vol. 22, pp. 503-504, 2015.

[131] Q. S. Han, "Clinical observation of 66 cases of chronic pelvic inflammatory diseases treated by hyperbaric oxygen combined with traditional Chinese and western medicine," 
Chinese Journal of Naut. Med High Press Medicin, vol. 23, pp. 239-240, 2016.

[132] Z. L. Zhang, "Therapeutic effect of hyperbaric oxygen combined with traditional Chinese and western medicine on pelvic inflammatory diseases," Med. Innov. China, vol. 6, p. 61, 2009.

[133] R. M. Xia, “"Clinical effect of microwave physiotherapy combined with Hongteng decoction and moxifloxacin in the treatment of chronic pelvic inflammation," Inn. Mong. Journal of Traditinal Chinese Medicine, vol. 39, pp. 105-106, 2020.

[134] J. J. Zhu, "The clinical effect of modified peony and licorice decoction retention enema combined with microwave physiotherapy on chronic pelvic inflammatory disease," Journal of Traffic Medicine, vol. 34, pp. 53-55, 2020.

[135] Y. X. Jia, Q. F. Meng, and M. P. Liu, "Clinical analysis of Hong Teng Tang retention enema combined with microwave physiotherapy in the treatment of chronic pelvic inflammatory disease," Chinese Community Dr.vol. 34, p. 106+108, 2018.

[136] Y. S. Jia, X. W. Zhang, and Z. K. Shi, “Clinical effect of kangfu anti-inflammatory suppository combined with microwave therapy on chronic pelvic inflammatory disease," Medical Recapitulate, vol. 24, pp. 3737-3740, 2018.

[137] T. Y. Duan, “"Discussion on the clinical effect of traditional Chinese medicine retention enema combined with microwave physiotherapy in the treatment of chronic pelvic inflammatory disease," Electron," Journal of Practical Gynecological Endocrinology, vol. 6, p. 117+120, 2019.

[138] S. W. Shi and L. Xu, "Treatment of chronic pelvic inflammation with traditional Chinese medicine retention enema combined with abdominal microwave and foot bath with traditional Chinese medicine," Mod. Regimen, vol. 188, 2016.

[139] L. Wang, "Effect of mild moxibustion and microwave therapy on chronic pelvic inflammatory disease with qi stagnation and blood stasis syndrome," Med. Equip, vol. 30, no. 11, pp. 132-133, 2017.

[140] T. Sha, Comparison of Nursing Effect of Mild Moxibustion Combined with Microwave Therapy on Chronic Pelvic Inflammatory Disease with Qi Stagnation and Blood Stasis, Heilongjiang University of Chinese Medicine, Harbin, China, 2015.

[141] Y. Wang, "Clinical observation on effect of warm acupuncture with microwave therapy for teating chronic pelvic inflammatory in 186 cases," Guangming Journal of Chinese Medicine, vol. 29, no. 11, pp. 2338-2339, 2014.

[142] L. T. Wu Gm and Y.Z. Chen, "Quantitative study on physical parameters of cupping therapy and its significance," Shanghai Journal of Acupuncture Moxibustion, vol. 40, pp. 890-893, 2021.

[143] "The ISO international standard 'traditional Chinese medicine-glass cupping tools' was published," Tianjin Journal of Traditional Chinese Medicine, vol. 40, p. 187, 2021.

[144] Z. Q. Xi Xd and H. Y. Ming, "Clinical study on the treatment of chronic pelvic inflammatory disease by "dragonfly-dipping water" acupuncture combined with cupping in abdominal sacral region," Shaanxi Journal of Traditional Chinese Medicine, vol. 40, pp. 1771-1773, 2019.

[145] L. Yj, "Clinical observation of bamboo tube cupping combined with blood prickling therapy for chronic pelvic inflammatory disease," Journal of Practical Traditional Chinese Medicine, vol. 33, p. 568, 2017.

[146] Z. Q. Liu, "62 cases of chronic pelvic inflammatory disease treated by acupuncture and specific electromagnetic therapy equipment combined with cupping," Chinese Journal of Modern Drug Application, vol. 42976, 2021.

[147] D. Đuričić, H. Valpotić, and M. Samardžija, "Prophylaxis and therapeutic potential of ozone in buiatrics: current knowledge," Animal Reproduction Science, vol. 159, pp. 1-7, 2015.

[148] A. Barone, M. Otero-Losada, A. M. Grangeat et al., "Ozonetherapy protects from in-stent coronary neointimal proliferation. Role of redoxins," International Journal of Cardiology, vol. 223, pp. 258-261, 2016.

[149] Z. Merhi, B. Garg, R. Moseley-LaRue, A. R Moseley, A. H Smith, and J Zhang, "Ozone therapy: a potential therapeutic adjunct for improving female reproductive health," Medical Gas Research, vol. 9, no. 2, pp. 101-105, 2019.

[150] A. Wei, H. Feng, X.-M. Jia, H. Tang, Y.-Y. Liao, and B.-R. Li, "Ozone therapy ameliorates inflammation and endometrial injury in rats with pelvic inflammatory disease," Biomedicine \& Pharmacotherapy, vol. 107, pp. 1418-1425, 2018.

[151] B. M. Escandón, J. S. Espinoza, F. P. Perea et al., "Intrauterine therapy with ozone reduces subclinical endometritis and improves reproductive performance in postpartum dairy cows managed in pasture-based systems," Tropical Animal Health and Production, vol. 52, no. 5, pp. 2523-2528, 2020.

[152] L. Z. Wei, Z. Jin, and L. H. Wei, "Guidelines for the clinical application of proprietary Chinese medicines in the treatment of sequelae of pelvic inflammatory diseases," Chinese Journal of Integrated Traditional and Western Medicine, vol. 41, pp. 286-299, 2021. 